\section{Detection of intergalactic gas in distant, rich clusters}

WE have detected a reduction in intensity or 'cooling' of the cosmic microwave background as this radiation passes through several rich clusters of galaxies. These observations imply the presence of substantial quantities of hot intergalactic gas in the clusters observed. The 'cooling' effect, due to inverse Compton scattering of the microwave photons by hot intergalactic gas in the clusters, was predicted earlier by Sunyaev and Zel'dovich ${ }^{1}$. The fractional change in the intensity of the microwave background is given by

$$
\frac{\Delta I}{I}=\frac{x \mathrm{e}^{\mathrm{x}}}{\mathrm{e}^{\mathrm{x}}-1}\left[\frac{x}{\tanh (x / 2)}-4\right] \int_{0}^{\tau} \frac{k T_{\mathrm{c}}}{m_{\mathrm{e}} c^{2}} d \tau \ldots
$$

where $x$ is related to the observing frequency by $x=h v / k T_{\mathrm{r}}$, with $T_{\mathrm{r}}$ the temperature of the microwave background, taken to be $2.7 \mathrm{~K} ; \tau$ is the total optical depth for Thomson scattering through a cluster; and $m_{\mathrm{e}}$ and $T_{\mathrm{e}}$ are the electron mass and electron temperature of the hot gas. Gull and Northover ${ }^{2}$, observing at $\lambda=3 \mathrm{~cm}$, have recently reported evidence for this effect in several clusters. We have tried to confirm their work at somewhat higher sensitivity, and at a shorter wavelength. A shorter wavelength was chosen to lessen interference and confusion by radio sources in or ncar the clusters observed. Our results on eight clusters which are Uhuru X-ray sources will be reported elsewhere ${ }^{3}$. In general, we were able to set $2 \sigma$ limits of $\lesssim 4 \times 10^{-4} \mathrm{~K}$ on the reduction in tcmperature of the cosmic background; in some cases these limits lie below the results reported by Gull and Northover. We do see statistically significant evidence for the 'cooling' effect in three clusters, however-all of which are in Abell ${ }^{4}$ richness class 4. In the case of one of the three, Abcll 2218, our results confirm the measurement of $\Delta T=-1.94 \pm 0.54 \mathrm{mK}$ by Gull and Northover ${ }^{2}$. Here we call attention to the apparent strong correlation between the richncss class and the detectability of the inverse

Table 1 Richness class 4 clusters observed. The measured changes in temperature, with their associated standard deviations of the mean, are given. The approximate values of the proton density in the clusters are derived under the assumptions listed in the text.

\begin{tabular}{ccccc}
\hline Abell clustcr & $\alpha_{1950}$ & $\delta_{1950}$ & $(\Delta T \mathrm{mK})$ & $\begin{array}{c}\text { Inferred } n_{9} \\
\left(10^{3} \mathrm{~cm}^{-3}\right)\end{array}$ \\
1689 & $13 \mathrm{~h} 08 \mathrm{~m} 58 \mathrm{~s}$ & $-01^{\circ} 06.5^{\prime}$ & $-1.06 \pm 0.46$ & $5 \pm 2$ \\
2125 & $15 \mathrm{~h} 40 \mathrm{~m} 26 \mathrm{~s}$ & $+66^{\circ} 28.8^{\prime}$ & $-3.10 \pm 0.34$ & $14 \pm 1.5$ \\
2218 & $16 \mathrm{~h} 35 \mathrm{~m} 43 \mathrm{~s}$ & $+66^{\circ} 19.5^{\prime}$ & $-2.65 \pm 0.23$ & $12 \pm 1$ \\
\hline
\end{tabular}

Compton 'cooling'. These results may be of interest to those constructing models for the intergalactic plasma in clusters ${ }^{5-11}$, especially when the results are combined with the more sensitive $\mathrm{X}$-ray observations we can expect if the new HEAO satellites are successful.

Our measurements were made at $\lambda=9 \mathrm{~mm}$ at the Cassegrain focus of the 11-m NRAO* telescope in Tucson, Arizona, during excellent weather in April 1977. The National Radio Astronomy Obscrvatory is operated by Associated Universities, Inc., under contract with the NSF.) At the time we used them, the dual receivers had system temperatures of $\sim 560 \mathrm{~K}$ (double sideband), with $\Delta v=10^{9} \mathrm{~Hz}$. The telescope beam was $3.6^{\prime}$ full width at halfpower; we employed beam switching in azimuth with a beam throw of $9.0^{\prime}$.

Three richness class 4 clusters were observed for periods of 5-9 h; 'cooling' of the microwave background was detected in all of them. The observed changes in temperature and the associated standard deviations of the means are shown in Table 1. All data were weighted equally in forming these means. The values shown in Table 1 have been corrected for atmospheric extinction, and for aperture and beam efficiency ${ }^{3}$, using a figure of $40 \%$. We note that each of these values of $\Delta T$ is larger in magnitude than any $\Delta T$ measured for nearby, but less rich, clusters such as Coma or Abcll 576.

Four corrections to the tabulated results arc necessary before they can be used to draw inferences about the amount and distribution of ionised matter in these clusters. First, the observed $\Delta T$ values are expressed in antenna temperature, and must be converted to differences in thermodynamic temperature for comparison with the $2.7 \mathrm{~K}$ background. The correction factor is 1.027. This reduction in the sensitivity of the results is more than off-set by an increase in the magnitude of the function of $x$ in equation (1). With these two corrections applied, we find

$$
\int_{0}^{\tau} \frac{k T_{\mathrm{e}}}{m_{\mathrm{e}} \mathrm{c}^{2}} \mathrm{~d} \tau=-0.15 \Delta T
$$

Next, the antenna pattern must be convolved with the surface brightness distribution of the source. The latter, of course, is model dependent. If we adopt, for instance, the adiabatic model of Sarazin and Bahcall ${ }^{5}$, with $T_{\mathrm{e}}=10^{8} \mathrm{~K}, H_{0}=55 \mathrm{kms}^{-1}$ per $\mathrm{Mpc}$, and a cluster core radius of $0.25 \mathrm{Mpc}$, we find the values of the average proton number density given in Table 1. Fourth and finally, a correction should be made for the finite separation of our beams $\left(9.0^{\prime}\right)$ compared to the cluster radii. For the Sarazin and Bahcall models, however, this correction can be neglected for these distant clusters.

The large size of the microwave effect and the inferred values of $n_{0}$ suggest that these clusters should be luminous $X$-ray sources ${ }^{11}$. That they are not included in the $4 \mathrm{U}$ catalogue is no doubt due to their distance: all are Abell distance class 6. Our results do suggest, however, that these rich clusters-despite their distance should be readily detected by the next generation of Xray satellites, and that they may prove to be among the most luminous $\mathrm{X}$-ray clusters.

GEORGE LAKE

Physics Department,

Princeton University,

Princeton, New Jersey

R. B. PAR'TRIDGE

Haverford College,

Haverford, Pennsylvania

Received 4 August; actepled 19 Octoher 1977.

I. Sunyacv, R. A. \& Zel'dovich, Ya. B. Commems Astruphis. Space Sti. 4, 173(1972)

2. Guti, S. F. \& Northover, K. J. E. Nalure 263, 572 (1976).

3. lake, (i. \& l'artridge, R. I3. Asrrophls. J. (submitted).

Abell, G. O. Astrophys. J. Suppl. 3,211 (1958).

5. Sarazin, C. L. \& Bahicall, J. N. Astrophys. J. Sulpl. 34, (1977).

Gull, S. F. \& Northover, K. J. E. Mon. Not. R. a.str. Soc. 173, 585(1975)

. Lea, S. M. Astrophy's. Lett, 16, 141 (1975).

Cavaliere. A. \& Fusco-Femiano, R. Astr. Astrophys, 49, 137 (1976).

. Bahcall, J. N. \& Sarazin, C. I.. Astrophys. J. Le'tt. 213, L99 (1977).

Gould. R. J. \& Rephacti, Y. Compron Scattering of Micronture Buch ground Rutimion by (ias in Galuxy Cluster's (to be published).

11. Bahcall, N. A., preprint (1977).

\section{Radio polarisation of quasars with optical absorption spectra}

CONWAY and Gilbert ${ }^{\lrcorner}$have noted that the mean linear polarisation at wavelength $\lambda 49 \mathrm{~cm}$ of highly redshifted quasars is significantly less for those with optical absorption lines compared with those with none. They suggested that the cause was Faraday depolarisation in the same regions responsible for the absorption lines. Gardner and Whiteoak ${ }^{2}$ pointed out similar differences between the two quasar types at $\lambda \dot{\lambda} 11 \mathrm{~cm}$ and $6 \mathrm{~cm}$. They regarded lower intrinsic polarisation of absorption-line quasars as a more likely explanation. Both findings were based on extremely small statistical samples. Subsequently much more data have become available at both optical and radio wavelengths. The significance of absorption-line quasars has also bcen enhanced. We therefore undertook a study using today's greater sel of data. 